\title{
Microcellular Foam Injection Molding Surface Improvement Methods
}

\author{
Hui-Lei LU ${ }^{\mathrm{a}}$, Guang-Hong HU \\ Institute of Forming Technology \& Equipment, Shanghai Jiao Tong University, 1954 Hua Shan \\ Rong, Shanghai, 200030, China
}

a67246135@qq.com, bhguangh@sjtu.edu.cn

Keywords: Microcellular, Foam Injection, Surface Defect, Surface Quality Improvement.

\begin{abstract}
Microcellular foam injection molding process is a new technique and widely used in different industries nowadays. However, most products have surface defects that limit this process usage. This paper introduces different methods used on improving microcellular foam injection molding parts surface quality. And each method theory is explained accordingly.
\end{abstract}

\section{Introduction}

The energy crisis and environmental pollution problems are guiding our industries to use foaming technique to create light weight material instead of solid material. The foamed material has taken its advantage by using less material and consuming less energy. Compared with solid material, foamed material has been widely used as insulator, structure part with stiffness, impact absorber, wood replacement, no-sink-mark appearance part and dimensional stable part. However, its large and non-uniform cell size has limited its usage with low mechanical strength. Nevertheless, microcellular techniques repossess the mechanical strength loss and lack of uniformity of the cells, and offer all the foamed technique advantage.

Microcellular foam injection molding process (MFIM) is a technique melt resin with supercritical fluids ( $\mathrm{SCF}$, e.g. $\mathrm{CO}_{2}$ or $\mathrm{N}_{2}$ ) to foam saturated solution ${ }^{[1]}$. Compared with conventional injection molding process, microcellular injection molding process can reduce product weight without losing much mechanical property. This is because it can reduce material and keep most of the stiffness. On the other hand, the tiny spherical cell in the resin can stop crack extension. These cells can buckle the crack tip and retard crack growth ${ }^{[2]}$. Furthermore, the microcellular structure will decrease shrinkage and warpage, thus improve dimensional stability of the product. Last, SCF always acts as a temporary plasticizer, resulting the SCF melt lower viscosity than pure polymer melt. Thus, related temperature and pressure requirements to injection machine are decreasing.

According to the above-mentioned advantages, the MFIM has been used in many industries such as automotive and electrical goods using a broad range of thermal plastics.

However, the microcellular foam injection products always have surface defects such as burst and dragged cell leading to streaking, swirling/elephant skin, and spooge/blood slug ${ }^{[3]}{ }^{[4]}$. Among the above deflects, swirling is the most common one and not easy to be removed. Scientists had taken good effort to study the mechanism of the swirl forming. And this paper is aim to introducing these methods, and explaining the merit and demerit for these methods.

\section{Principle}

\section{Supercritical Fluids}

The SCF is the gas in a certain pressure and temperature higher than the critical pressure and temperature. The shadowed area in Figure 1 represents the temperature and pressure region of SCF state $^{[3][5]}$. In this state, the gas is in its liquid like state, or on other word, the liquid is in its gas like state. In fact, it has both gas-like and liquid-like properties. The gas-like property mainly represents the low viscosity similar to air viscosity, although it's several times higher than the gas phase. The 
liquid-like property is that the density is heavy compare to gas density, and its density value is much more close to liquid.

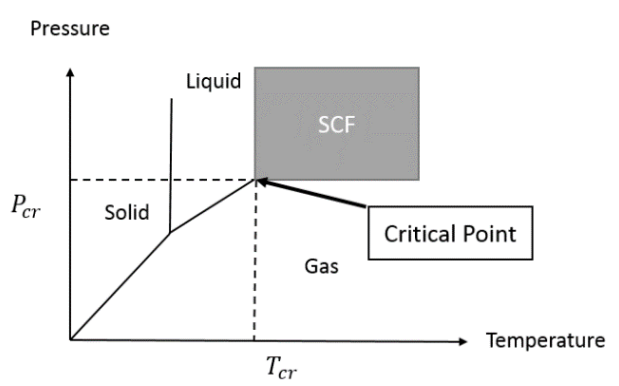

Fig.1 Diagram of Material Phase

\section{Microcellular Foam Injection Molding Process}

Microcellular foam injection molding process involves four steps: gas dissolution, nucleation, cell growth, and shaping ${ }^{[6]}$. In gas dissolution stage, The SCF is metered and injected into barrel with a special screw, which is designed to maximize the mixing and dissolving of the gas. Melt polymer and the SCF are mixed. During injection, the nucleation sites foamed due to the rapid and substantial pressure drop in the mold. The main mechanism of nucleation is thermodynamic instability caused by pressure drop. During the rest of injection, the cells continue to grow from the nucleation site formed in formal stage, and fill the mold. The growth is influenced by processing conditions such as melt pressure, temperature, material properties such as resin viscosity, melt strength and gas solubility, mold structure such as foaming space in the cavity. Finally, the part shaped and subsequently ejected after the mold cooling process.

\section{Swirl Mechanism}

There're two mechanisms to explain how this defect formed. One is broken bubble from the freeflow front, which is well known as "fountain effect","7] "Fountain effect" says the cells in the center of the flow continue growing at mold filling stage. And if the mold filling time is long, the cell will break to form "free-flow front roughness". This kind of roughness only become part of surface roughness, most time at the edge of the part far away from the gate. (Figure 2) It is because the flow at this position suffers longest filling time and the cells most likely growing to break. So the "fountain effect" can also be used as a control factor for the maximum limit of injection time.

The other mechanism is sheared bubble in the interface between mold wall and melt, it is called as "interface roughness" [7] "Interface roughness" illustrates that the cell condition is at the edge of the flow. If the adhesion or friction force is high, the cell at the interface will have zero velocity. The cell will deform to a spherical shape under the friction force, and even become flattened in the most shearing field. Some cells even tear to break and these cells form the roughness and swirl streak (Figure 3) ${ }^{[3]}$. This swirl streaking is always close to gate where cells suffer the highest friction force. Sometime even far away from the gate, there's a compelling flow under a low temperature and still high shear rate condition on skin layer, swirl surface is still formed.

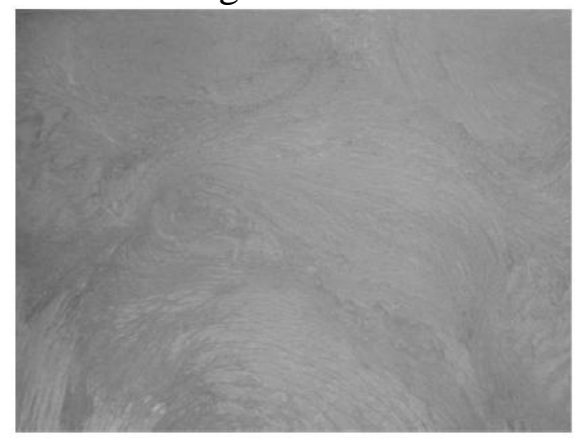

Fig.2 Swirl Surface for Fountain Effect ${ }^{[8]}$

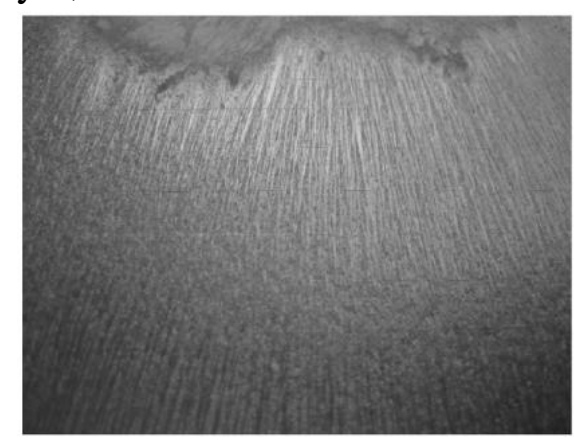

Fig.3 Swirl Streak for Interface Roughness ${ }^{[8]}$ 


\section{Methods to Improve Surface Quality}

Different methods are used to improve surface quality of microcellular foam injection molded parts. There are two main approaches. One is to control temperature, and the other is to control pressure. Both share a fundamental thought as to keep SCF from nucleation. Besides, there're other methods like directly retard cell nucleation, or use co-injection to make a "sandwich" structure. The methods will be listed and explained one after another.

\section{Active Temperature Control}

The actively control mold temperature method was first developed by Trexel and One Sangyo ${ }^{[9]}$. Trexel and One Sangyo had joint developed a new technology named MuCell Gloss. It combined Trexel's microcellular technique and One Sangyo's rapid heat cycle molding technique (RHCM). RHCM is a technique adding two fully independent temperature control circuit to the system. One circuit is to keep surface above heat distortion temperature during fill stage, the other is to achieve rapid cooling once mold filling is completed. This technique opens the door for high-surface-quality microcellular foam injection part.

The main principle of active temperature control method is to eliminate bubble by heating the mold. The reason is that hot mold has less friction or adhesion between cavity wall and melt. So that the flow is acting like a plug flow. For plug flow, the theoretical velocity distribution is equal in the thickness direction. Thus there's no shearing between flow layers in the depth direction. Furthermore, the breaking bubble from a free surface will be ironed or repaired by hot surface under cavity pressure $^{[3]}$. Even to crystalline material, this technique gives enough time for crystallization and gas diffusion back into skin.

Cha analyzed the cause of surface swirl marks as a function of mold temperature ${ }^{[10]}$. A model was used to explain how swirl marks appear. Inside the cavity, the temperature in the center of the cavity was almost equal to melt temperature, and the temperature close to cavity interface was equal to mold temperature. (See Figure 4.) When the solution stream at fountain front reached the cavity wall, the gas generates due to the temperature and pressure decrease. At the same time, the plastics/gas solution was solidified since mold temperature was always below polymer glass transition temperature (or crystal temperature of crystalline polymer). So the gas bubbles stretched in the solution turbulence flow had form a plot and the plot had been "freeze" in the resin mold interface. That was called as swirl marks on the part surface. According to that, Cha got the idea that if plastics/gas solution wasn't solidified when it contacted the mold surface, the gas bubble from the solution would be forced to flow from the surface to the cavity end.

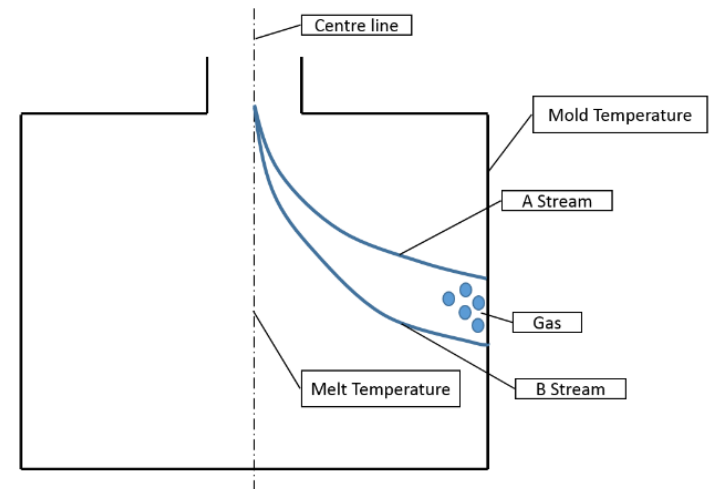

Fig.4 Mechanizon for Swirl Mark

Cha did some experiments controlling the mold temperature by installing heat cartridge and thermal couple at cavity. And he figured out a critical temperature which had obvious effect for surface improving. PP (crystalline material) and ABS (amorphous material) were used as base resin. The results of the experiments showed that both PP and ABS had swirl marks disappeared at a mold temperature higher than the typical temperature. 
According to Yoon and Cha's research ${ }^{[10]}$, the glass transition temperature decreased because the dissolved gas lowers the viscosity of the polymer. It explained why the swirl disappear temperature decreased. And finally Cha got the conclusion that swirl marks could disappear when mold temperature is higher than the reduced glass transition temperature. There is something special for crystallization material. It is because higher temperature will cause more crystallization, and more crystallization on hot mold surface will create high non-uniformity of cell structural. Thus rough surface is caused as well. So for crystallization material, there's a balance between crystallinity and small cell structure ${ }^{[3]}$.

\section{Passive Temperature Control}

Another work is to add the coated insulation film on the mold surface. It significantly reduces the friction between melt and mold surface and the shearing as well.

Chen Hui-Li etc. ${ }^{[11]}$ stick thermally insulated polymer film, composite with $82 \%$ PET and $18 \%$ PC, on the mold core surface to achieve heat transfer delay effect. The swirl marks and silver streaks were eliminated when the thickness of the film reach $0.188 \mathrm{~mm}$. At that time, the surface roughness of the product is $1.8 \mu \mathrm{m}$. The theory was the same as active mold temperature control method. The insulator reduced shearing between mold and melt, and kept mold temperature high enough to iron and repair the bubble on the surface.

Turng and his group ${ }^{[12]}$ had inserted PTFE film on the mold; they also investigated the thickness of film effect, and got the conclusion that $175 \mu \mathrm{m}$ thickness was enough to eliminate the surface swirl marks.

The hot mold surface method fixed only one aspect of the swirl defects, "shearing" one. It had no effect on the "fountain flow" effect. Furthermore, for crystalline materials, the higher mold temperature would create higher non-uniformity of crystallinity, so there should be a balance between crystallinity and cell structure ${ }^{[16]}$.

\section{Gas Counter Pressure}

A sealed mold charged with gas counter pressure (GCP) prior to the injection is also a good method for surface finish improvement ${ }^{[13-14]}$. A critical melt flow front pressure was used to eliminate surface swirl marks. The GCP microcellular part always display more uniform cell structure, and then result in improving part physical property.

Unlike temperature methods, the idea to control SCF Pressure could fix both "fountain flow" defect and "interface roughness" defect. In most documents, the theory of gas counter pressure could be explained by energy theory ${ }^{[17-18]}$. The main aim of the energy theory is to improve surface finish by nucleation rate decreasement at filling stage when the flow is disordered. The nucleation is delayed till the flow calm down.

According to classic nucleation theory, the nucleation rate is controlled by macroscopic properties such as solubility, diffusivity, surface tension, gas concentration, temperature, and the degree of supersaturation ${ }^{[15-16]}$. Colton and Suh reported a representative equation for nucleation rate of polymeric foams ${ }^{[17-18]}$. The equation can be expressed as

$$
N=f_{0} C_{0} \exp \left(\frac{-\Delta G^{* *}}{k T}\right) \text {. }
$$

Where $N$ is the nucleation rate, $f_{0}$ is the frequency of atomic molecular lattice vibration, $C_{0}$ is the concentration of gas molecules, $\mathrm{k}$ is the Boltzmann's constant, $T$ is the absolute temperature, and $\Delta G^{* *}$ is the activation energy barrier for nucleation.

If the nucleation is considered ideally homogeneous, the $\Delta G^{* *}$ can be express as

$$
\Delta G^{* *}=\Delta G_{\text {hom }}^{*}=\frac{16 \pi}{3 \Delta p^{2}} \gamma_{b p}^{3} .
$$

Where $\Delta G^{* *}=\Delta G_{\text {hom }}^{*}=\frac{16 \pi}{3 \Delta p^{2}} \gamma_{b p}^{3}$ is the critical free energy barrier for homogeneous, $\Delta G^{* *}=\Delta G_{\text {hom }}^{*}=\frac{16 \pi}{3 \Delta p^{2}} \gamma_{b p}^{3}$ is the surface energy of the polymer-bubble interface. So $\Delta \mathrm{p}$ is the 
difference between the gas pressure inside the nucleus or bubble and the melt pressure outside the nucleus.

$$
\Delta p=\left|p_{r}^{\prime}-p_{r}\right| \cdot
$$

$p^{\prime}{ }_{r}$ is the pressure that is exerted in a high pressure vessel and $p_{r}$ is the pressure of supersaturated vapor in the sample. When gas counter pressure increase, $\Delta p$ will decrease, and $\Delta G^{* *}=\Delta G_{\text {hom }}^{*}=\frac{16 \pi}{3 \Delta p^{2}} \gamma_{b p}^{3}$ increase. With the increase of the $\Delta G^{* *}$, nucleation rate decreased. It meant that more energy was needed for the cell to reach rupturing energy barrier. So when gas counter pressure was inserted into the cavity, the nucleation had been hold-up until the pressure released. That's the energy theory explained how gas counter pressure affected surface quality.

The process of gas counter pressure can be described as the following: The sealed mold closed, and a valve opened to pressurized mold cavity to a certain pressure environment. The specialized pressure should be higher than foaming pressure but lower than injection pressure. Please noted that although the regular gas counter pressure required the gas pressure to be larger than the foaming pressure, thus additional $25 \%$ to $100 \%$ clamp tonnage was needed comparing to structural foam depending on venting technique used ${ }^{[19]}$. However, microcellular molding might allow a much lower restriction pressure. Especially for short shot method mentioned above. The subsequently injected microcellular melt resin would be suppressed from cell nucleation by high pressure environment. At the end of injection, the counter gas pressure would be released and the nucleation began. Since there's no cell during injection, the fracture of the cell on the melt front was inhibited, thus the gas flow marks was decreased or eliminated.

Shuai Li etc, did an analysis to describe the GCP effect on part surface quality ${ }^{[20]}$. He used a commercial grade HIPS (PH-888G) polymer as injection material. Nitrogen was used as physical blowing agent. He proved that both GCP and holding time increasing could have swirl marks decreasement effect. And the holding time effect was less than GCP on surface gloss.

$\mathrm{Xu}$. J did an experiment on clear PC material, the experimental results shown that due to the pressure drop rate reduced with gas counter pressure molding, the number of cells was less than that in normal forming ${ }^{[21]}$. And the parts weight was reduced from $8 \%$ to $24 \%$ compared with gas off sample. On the other hand, the resistance of gas pressure in the cavity kept cells in the spherical shape and more uniform from gate to the end.

Lee et al. also presented the result of the gas counter pressure with mold crack opening method for various PP materials ${ }^{[22]}$. The results were similar with PC materials that the cell density was obviously lower although the cell uniform was much better.

A special gas counter pressure from Engel had supplied a gas recovery system ${ }^{[23]}$. The pressure in the mold could be controlled just high enough to keep the gas in the single-phase solution still in the solution on the flow front. This prevented the creating of surface swirls. The material used was an unreinforced grade of Bayer's Markrolon PC. The surface roughness improved from $23.11 \mu \mathrm{m}$ to $0.85 \mu \mathrm{m}$ compared with regular microcellular PC parts.

\section{Co-injection}

Co-injection technique was invented by Garner and Oxley of $\mathrm{ICI}^{[24]}$, and has been used since 1970s. It's used not only for a perfect smooth surface, but also for part property improvement or for economic recycling parts. The microcellular co-injection part usually has a non-foamed skin with a microcellular core.

The sequential process is skin-core-skin with three steps. The first step is to inject the skin material about $25 \%-40 \%$ of full shot size to form enough flow front and frozen skin on the wall. The core material is injected secondly when the skin material in the center remains molten. The core material can be injected purely or with a center percent of skin material on this stage. Finally, the core material stops being injected and the skin material is injected in to the mold again to seal the gate area by skin. And thus, the core material is cleaned in the runner and nozzle channel with skin being the only material for the next shot. 
In co-injection, the main threaten is that core material penetrate the skin. Studies showing that the combination of low-viscosity core and high-viscosity skin caused more core breakthrough defect ${ }^{25-}$ ${ }^{27]}$. This defect was called core surfacing, or core breakthrough ${ }^{[28-30]}$.

Han did some experiments with two different viscosity materials in the co-extrusion die ${ }^{[31]}$. One was polystyrene (PS), and the other was high-density polyethylene (HDPE). At temperature $200^{\circ} \mathrm{C}$, the viscosity of PS was higher than HDPE. Then, the high viscosity PS was wrapped by lowviscosity HDPE. At temperature $240^{\circ} \mathrm{C}$, the viscosity of HDPE was higher than PC, thus, the highviscosity HDPE was wrapped by low viscosity PS. Therefore the conclusion was come that the lowviscosity material was always trying to first wet the surface.

Turng and Kharbas analyzed the penetration behavior as well ${ }^{[32]}$. A general-purpose polystyrene (GPPS) resin (BASF145D) was used as both skin and core material. They got the conclusion that the less viscous core material could only displace the skin material near the center of the part when the skin material remained hot ant fluid.

Turng et al. had applied co-injection technique into microcellular injection molding. The final part had a solid skin and foamed core ${ }^{[33]}$. However, the production cost is very expensive. The cost hindered the popularization of this technology.

\section{Control the Cell Nucleation Rate}

Lee et al. used the method to improve surface quality by controlled cell nucleation rate and reduced cell concentration. By controlling the degree of super saturation, the nucleation delayed during the filling stage ${ }^{[4]}$.

He added the degree of supersaturation factor in Colton's energy theory.

$$
\Delta p=\left|p^{\prime}{ }_{r}-p_{r}\right|=\frac{2 \gamma}{r_{c}} \text {. }
$$

where $r_{\mathrm{c}}$ is the radius of a characteristic droplet, and yield by W.Thomson Equation.

$$
\operatorname{RTIn} \frac{p_{r}}{p_{\infty}}=\frac{2 \gamma^{* M}}{r_{c^{*} \rho}} .
$$

where $p_{\infty}$ is the pressure of the saturated vapor, $\mathrm{R}$ is the universal gas constant, $\mathrm{M}$ is the molar mass, and $\rho$ is the density. The equation yield:

$$
\Delta P=\frac{R T I n \frac{p_{r}}{p_{\infty}}}{M} .
$$

Finally the activation energy equation for nucleation in microcellular injection molding process can be given by:

$$
\Delta G_{\text {hom }}^{* *}=\frac{16 \pi}{3\left(k T \rho_{l} I n S\right)^{2}} \gamma_{b p}^{3} .
$$

And the conclusion is that the activation energy for nucleation was inversely proportional to the square of the natural logarithm of the super-saturation degree.

The project obtained a swirl-free microcellular injection molded part. The advantage was that this technique didn't need additional equipment. But it had limitation that low weight percentage of foaming agent, which was not favorable to weight reduction of products, must be adopted.

\section{Conclusion}

According to the above introduction, the possible methods for improving microcellular surface quality are summarized as follows:

There are two main theories for swirl problem, "surface roughness" and "fountain flow". The control mold temperature method, whether actively control or passively control by adding films, would only solve "surface roughness" swirl problem. The gas counter pressure and control cell nucleation methods could solve both "surface roughness" and "fountain flow" swirl problem. Both methods could explain by energy theory. However, the gas counter pressure method needed very complex and expensive additional equipment. Although the control cell nucleation method didn't 
need extra equipment, it had limitation on material resin selection. On processing side, adjusting processing parameter was an effective way to improve surface defect. But there's no systematic theory or guide booklet on how to process the adjustment. Different companies used their own experience on their focused products.

\section{Acknowledgement}

The authors would like to thank Shanghai Nature Foundation, China (No.13ZR1420500) for financial support.

\section{References}

[1] A. O. Herausgegeben, L.S. Turng, P. Gramann, Injection Molding Handbook, M, 2008.

[2] N.P. Suh, Innovation in Polymer Processing, M. Amer Society of Mechanical Engineers, 1982.

[3] J. Xu. Microcellular Injection Molding, M. 2010.

[4] J. Lee, LS. Turng, E. Dougherty, et al. A novel method for improving the surface quality of microcellular injection molded parts, J. Polymer, 2011, 52(6), pp. 1436-1446.

[5] Information on http://en.wikipedia.org/wiki/Supercritical_fluid

[6] Information on http://www.trexel.com

[7] J. Xu. Methods to the smooth surface of microcellular foam in injection molding, C.ANTEC CONFERENCE PROCEEDINGS, 2007, 4, pp. 2077.

[8] S.C. Chen, H.M. Li, S.S. Hwang, et al. Passive mold temperature control by a hybrid filmingmicrocellular injection molding processing, J. International Communications in Heat and Mass Transfer, 2008, 35(7), pp. 822-827.

[9] G. Eason, N. Benjamin, I.N. Sneddon. On certain integrals of lipschitz hankel type involving products of bessel functions, J. Philosophical Transaction of the Royal Society of London A: Mathematical, Physical and Engineering Sciences, 1966, 247(935), pp. 529-551.

[10] J.D. Yoon, S.W. Cha. Change of glass transition temperature as a function of gas, J. Polymer Testing. 2011, 20(3) pp. 287-293.

[11] H.L. Chen, R.D. Chien, S.C. Chen. Using thermally insulated polymer film for mold temperature control to improve surface quality of microcellular injection molded parts, J. International Communications in Heat and Mass Transfer. 2008, 35(8) pp. 991-994.

[12] J. Lee, L.S. Turng. Improvement in surface quality of microcellular injection molded parts, J. ANTEC2009, University of Wisconsi-Madison, Madison, WI, 2009, pp. 1662-1666.

[13] K.B. Andrzej, K. Hendrik, et al. Polycarbonate microfoams with a smooth surface and sigher notched impact strength, J. Journal of Cellular Plastics 2004, 40(6), pp. 489-496.

[14] K.B. Andrezej, F. Omar, K. Hendrik, et al. Microcellular polymer and composites, J. Polimery 2006, 51(10), pp. 697-703.

[15] L. Ari, T. Vicente, W.O. David. Nucleation: measurements, theory and atmospheric application - Annual Reviews, J. Annual Review of Physical Chemistry 1995, 46(1), pp. 489-524.

[16] C. Emo, S. Roberto. Biodegradable polymers and plastics, J. Springer; 2002.

[17] JS. Colton. Making microcellular foams from crystalline polymers, J. Plastic Engineering. 1988, 43(8), pp. 53-55. 
[18] JS. Colton JS, NP. Suh. The nucleation of microcellular thermoplastic foam with additives: part 1: theoretical considerations, J. Polymer Engineering and Science 1987, 27(7), pp. 485-492.

[19] S. Ham. Fundaments of structural plastics, J. Plastics Engineering. SPE publisher, 2001, 57(6), pp. 46-57.

[20] S. Li, G. Zhao, G. Wang, et al, Influence of relative low gas counter pressure on melt foaming behavior and surface quality of molded parts in microcellular injection molding process, J. Journal of Cellular Plastics. 2014, 50(5), pp. 415-435.

[21] J. Xu, Methods to the smooth surface of microcellular foam in injection molding, J. SPE ANTEC Tech. Papers, 2007, pp. 2089-2093.

[22] J. Lee, W.S. Wang, et al, Development of structural foams with a class-A surface using gas counter pressure and mold opening, J. CSME Forum Paper No. 1569101377 (June 2008).

[23] R. Neilley, Smoothing the surface finish of microfoamed parts, J. Injection Molding, 2003, 11(10), pp. 98-101.

[24] P.J. Garner, D.F. Oxley, British Paten No. 1, 156, 217 (1971).

[25] J.L. White, H.B. Dee. Flow visualization for injection molding of polyethylene and polystyrene melts, J. Polymer Engineering \& Science, 1974, 14(3), pp. 212-222.

[26] S.S. Young, J.L. White, et al. A basic experimental study of sandwich injection molding with sequential injection. [J] Polymer Engineering \& Science, 1980, 20(12), pp. 798-804.

[27] R.C. Donavan, K.S. Rabe, et al. Recycling plastics by two-shot molding, J. Polymer Engineering \& Science, 1975, 15(11),pp.774-780.

[28] L.S. Turng. Special and emerging injection molding proceed, J. Injection Molding Technology. 2001, 5(3), pp. 160-179.

[29] H. Eckardt, Co-injection charting new territory and opening new markets, J. Journal of Cellular Plastics 1987, 23(6), pp. 555-592.

[30] S. Ham. Fundamentals of structural plastics, J. Plastics Engineering. 2001, 57 (6), pp. 46-46.

[31] C.D. Han, Rheology in polymer processing, M. Academic Press, 1976.

[32] L.S. Turng, K. Hrishikesh. A novel microcellular co-injection molding process, C. ANTE conference proceedings. Society of Plastics Engineers, 2004, 1, pp. 535-539.

[33] L.S. Turng, H. Kharbas. Development of a hybrid solid-microcellular co-injection molding process, J. International Polymer Processing, 2004; 19(1), pp. 77-86. 